\title{
PEUTZ-JEGHERS SYNDROME: A CASE REPORT AND LITERATURE REVIEW
}

\author{
ISLAM MR ${ }^{1}$, HOSSAIN MS ${ }^{2}$, SHEIKH MSH ${ }^{3}$, RAHMAN MR ${ }^{4}$, LIMA IJ $^{5}$, KHATUN SA ${ }^{6}$, KASHEM MA ${ }^{7}$
}

\begin{abstract}
Peutz-Jeghers syndrome is characterized mainly by the presence of hamartomatous polyposis of gastrointestinal tract and melanin spots on the lips and buccal mucosa. The polyps are mostly found in the small bowel and less frequently seen in the stomach and colon. Intestinal polyps in short term may cause chronic bleeding, intestinal occlusion and intussusceptions. There is small but significant risk of small bowel adenocarcinoma, cancer of pancreas, breast, ovary and endometrium. A case of peutz-jeghers syndrome was presented with a complaint of episodes of colicky abdominal pain and vomiting; small bowel intussusception was diagnosed and an operation was performed in Colorectal Surgery unit of Bangabandhu Sheikh Mujib Medical University (BSMMU) Hospital, Dhaka, jejuno-ileo-caecal polyps were removed by enterotomy. On discharge she was instructed to attend our unit four weeks later for colonoscopic removal of sigmoid polyps and also advised to attend breast and cervical screening program.
\end{abstract}

Key words: Peutz-Jeghers syndrome, polyp, GIT.

J Dhaka Med Coll. 2014; 23(1) : 147-150.

\section{Introduction}

The first case of Peutz-Jeghers Syndrome (PJS) was presented by Hutchinson in 1896, when he described a case of twins with diffuse labial pigmentation. Y. Peutz, a Dutch pediatrician, in 1921, presented a case of intestinal familial polyposis associated with hyperpigmentation of skin and mucosa in general. In 1949, Jeghers et al. complemented the association of pigmentation and intestinal polyposis and confirmed the familiar origin of the disease. Since then, those features together were denominated Peutz-Jeghers Syndrome for this

1. Dr. Md. Rashidul Islam, Resident, MS (Phase-B), Department of Colorectal Surgery, Bangabandhu Sheikh Mujib Medical University (BSMMU), Dhaka.

2. Prof. Md. Shahadat Hossain, Department of Surgery, Bangabandhu Sheikh Mujib Medical University (BSMMU), Dhaka.

3. Prof. Md. Shahadot Hossain Sheikh, Department of Colorectal Surgery, Bangabandhu Sheikh Mujib Medical University (BSMMU), Dhaka.

4. Dr. Md. Rayhanur Rahman, Department of Colorectal Surgery, Bangabandhu Sheikh Mujib Medical University (BSMMU), Dhaka.

5. Dr. Ismat Jahan Lima, Department of Colorectal Surgery, Bangabandhu Sheikh Mujib Medical University (BSMMU), Dhaka.

6. Dr. Shima Akter Khatun, Assistant Professor, Department of Pharmacology, Dhaka National Medical College, Dhaka.

7. Dr. Md. Abul Kashem, Assistant Professor, Department of Medicine, Dhaka Medical College, Dhaka.

Correspondence: Dr. Md. Rashidul Islam, Resident, MS (Phase-B), Department of Colorectal Surgery, Bangabandhu Sheikh Mujib Medical University (BSMMU), Dhaka. Cell Phone: +8801711279298. disorder $_{1-5}$. PJS is an inherited, autosomal dominant disorder characterized by mucocutaneous pigmentation and gastrointestinal polyps ${ }_{2,3,5}$. Melanic spots are the earliest manifestation of PJS, typically appearing in the first year of life. Although most of the polyps reside in the jejunum, it may also occur in ileum,stomach, duodenum and/ or colon. Those polyps are present from childhood and may sometimes lead to intussusception or gastrointestinal bleeding 6 8. Carcinoma of GIT, pancreas, breast, ovary testicles and endometrium appear to be an infrequent complications of Peutz-Jeghers syndrome. By early diagnosis, prompt treatment, continuous follow up, and appropriate screening mortality and morbidity of PJS can greatly be reduced ${ }_{9-11}$.

\section{Case report}

An 18-year-old young girl has been referred in our unit from gastrointestinal department with a long history of intermittent abdominal pain and vomiting. The pain was somewhat diffuse and colicky in nature, without a precise localization and any specific causative factor. She also complained of vomiting and an evidence of per rectal bleeding. She has pigmented lesions of the perioral and perinasal skin, on the lower lip as well as hands and digits since birth and gradually increased .According to family history her father had also same type of pigmentation and died of stomach cancer. 


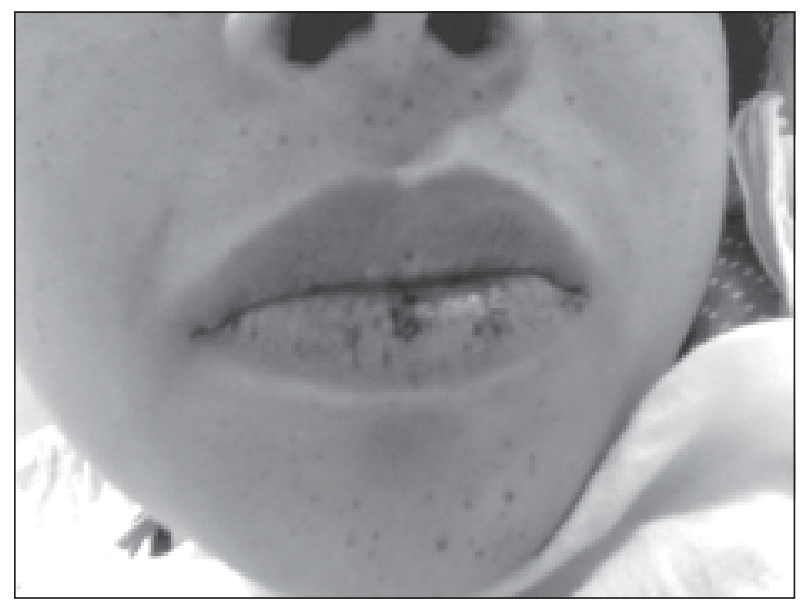

Fig.-1

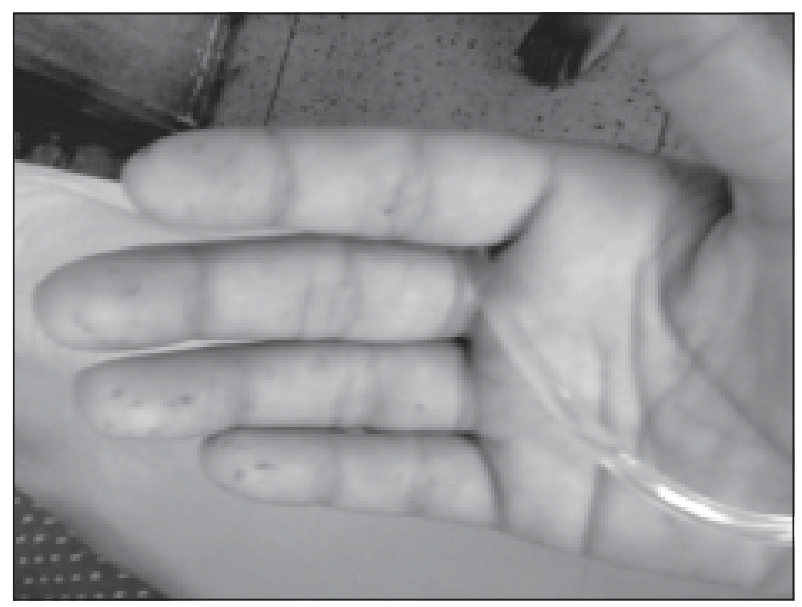

Fig.-2

On examination, the girl was found anxious, ill looking and found mild anemic. Dark pigmented maculae were present on perioral and perinasal skin, on the lower lip as well as hands and digits. Abdominal examination

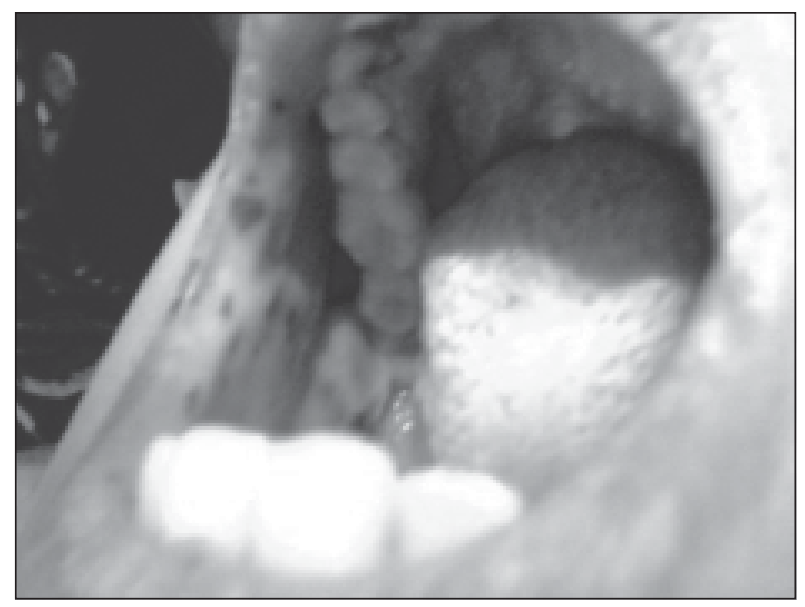

Fig.-3

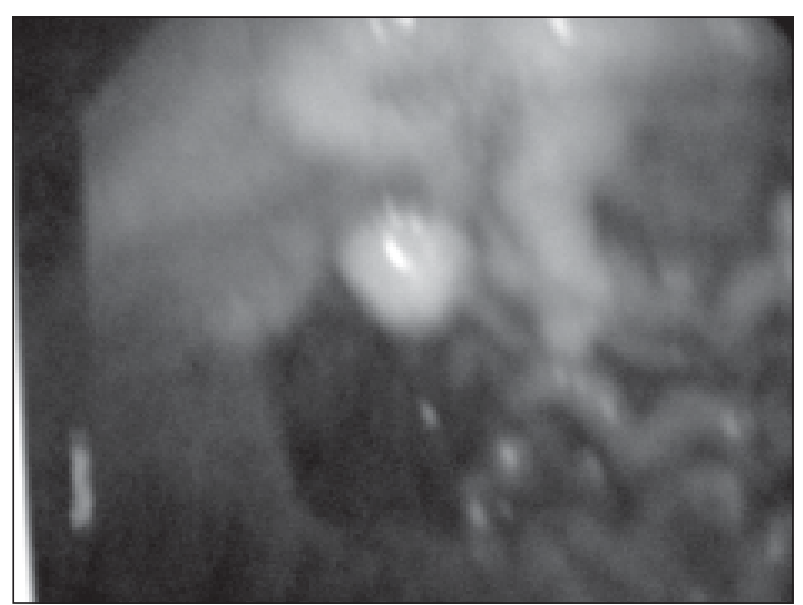

Fig.-4

reveals tenderness all over with an, ill-defined lump in left ileac fossa which disappears on next day on conservative treatment. No organomegaly were observed at physical examination. Abdominal ultrasound showed

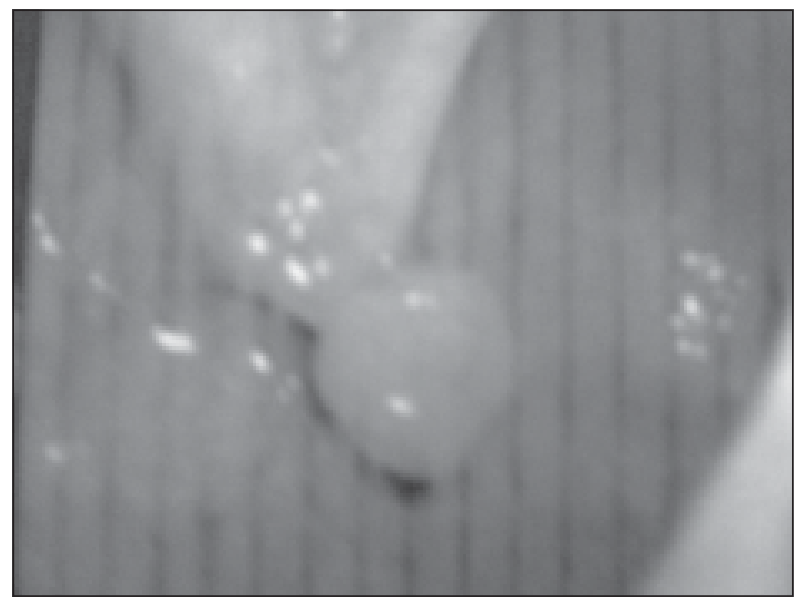

Fig.-5

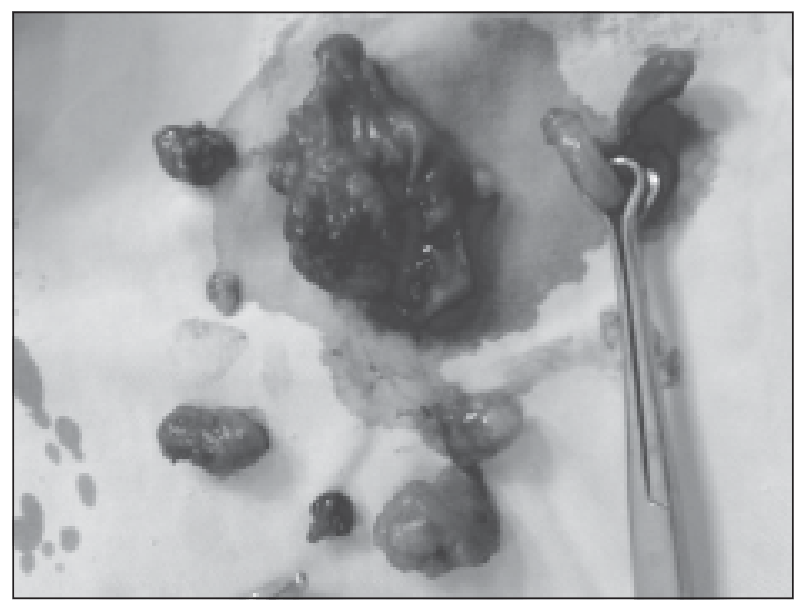

Fig.-6 
long segment circumferential colonic thickening and CT-scan revealed colonic thickening with irregular inner margin. Laboratory check-up was also within the normal range except mild normocytic normochromic anemia, upper digestive endoscopy discovered polyps in the antrum, body and fundus of stomach, and colonoscopy showed multiple polyps in the colon. At laparotomy three polyps in small intestine two in caecum and several small polyps were felt in sigmoid colon on digital survey, ileal, jejunal and caecal polyps were removed by enterotomy, while one large polyp from small intestine needs resection and anastomosis. Small polyps in sigmoid colon were left for later colonoscopic snaring. Tube caecostomy was done by foly's catheter and abdomen was closed after keeping a drain tube in pelvis.

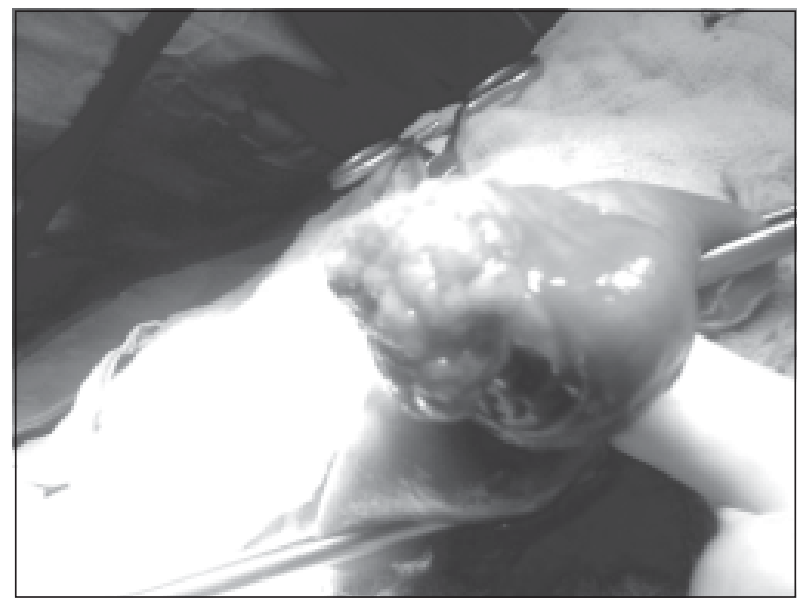

Fig.-7

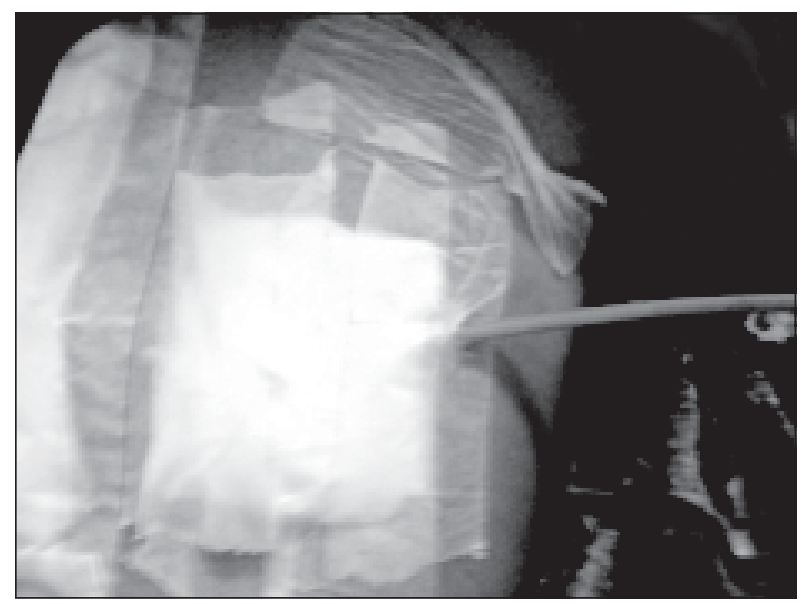

Fig.-8

\section{Discussion}

PJS is an autosomal dominant inherited syndrome consisting of gastrointestinal hamartoma and mucocutaneoushyper pigmentation, having an estimated prevalence of 1 in 120000 live births ${ }^{1,2}$. There is no sex or race predilection. The evolution of PJS during life exposes the patient to a predisposition to developing malignant neoplasias, mainly in the small bowel, caecum, rectum, as well as pancreatic adenocarcinomas. Other more rare carcinomas could occur in the breast, uterus, testicles and ovaries. The risk for cancer of those patients is 18 times higher in comparison with the general population, and as high as the number of intestinal polyps the higher is the chance for malignant transformation in PJS. The Involved chromosome is $19 \mathrm{p} 13.3$ with apparent mutation in the locus (STK11) LKB1 with the enzyme serin/treonin kinase functionally inhibiting the tumor suppressor genes ${ }^{1-3,10}$. The melanotic pigmentation could be present at birth or appearing later in childhood, with characteristic distribution in the perioral mucosa $_{2,11}$. It is important to emphasize that they are spots-like and not plaques, with alteration in color but not in the surface of the lesions. In the mouth, the main area of occurrence is the labial mucosa as well as the buccal mucosa, but they can also be found on the tongue, gingiva and hard palate, and are variable in number as well as in shape. In the skin they occur in general on the dorsum of the hands and feet, and also on the fingers. The lesions are very dark or dark blue, with a diameter ranging from 1 to $12 \mathrm{~mm}$. When the melanotic spots are localized on the face, the diameter of the spots rarely are larger than 2 $\mathrm{mm}^{1-3,10}$. The skin lesions could disappear during puberty but the lesions in the mouth persists forever. Histological evaluation show higher amount of melanin in the basal layer of the epithelium close to the basal lamina. The hamartomatous polyps are the main characteristic of the syndrome and they could vary in their shape, size, number and anatomical distribution, being found in different areas of gastrointestinal tract. In older patients, during colonoscopy multiple polyps are 
mainly found in the distal bowel region. The polypus could be sessiles or pedunculated, with a diameter varying between 1 to $3 \mathrm{~cm}$, and they could be considered to be capable to transformation in real cancer depending on the histological aspects seen in microscopy $2,7,9,12$. Approximatelly $35 \%$ of the patients with PJS were diagnosed in childhood or during puberty. In general they complain of abdominal pain, blood in the feces and alternating diarrhea and constipation. The symptoms of intestinal obstruction and/or hemorrage start to appear at 20 and 30 years of age ${ }^{7,9}$.

When there is a suspicion of PJS, the gastrointestinal tract of the patient needs to be evaluated and examined by gastric endoscopy and colonoscopy. The histological examination of the oral pigmentation includes large amounts of melanin in the basal layer of the epithelium. Histopathologically, the polyps of Peutz-Jeghers Syndrome are composed of normal elements indigenous to the site in which they arise, but in which the architecture is markedly abnormal. The lesions usually have an arborzing pattern with fronds covered by normal epithelium that incorporates all the cell types usually located in the site of origin. Endocrine cells are present in the polyps of all sites. Smooth muscle forms an important component of the lesions and extends into the branching fronds of the polyps. Benign glands may be surrounded by smooth muscle and may extend into the submucosa ${ }^{1}$.

The gastrointestinal surgeon together with the gastroenterologist is the indicated professional to treat the disease more suitably. Treatment depends upon the presentation. In case of intussusception or intestinal obstruction, surgical intervention is usually required. In patients with abdominal pain or gastrointestinal bleeding, radiographic imaging, endoscopy, or even virtual colonography may allow discovery of polyps that can be resected either endoscopically or by enterotomy, depending on their site and size. Advances in capsule and double-balloon endoscopy have markedly improved surveillance and management of polyposis of the small intestine. It is even possible to treat benign hyperpigmentation of the face by means of laser therapy. Q-switched lasers are the preferred method. The treatment is effective, but this is only complementary cosmetic therapy ${ }^{1,2}$.

\section{Conclusion}

Lentigines on the lips, is the sine qua non and, should alert the clinician to Peutz-Jeghers syndrome. As these patient are at increased risk of developing gastrointestinal and other extra intestinal malignancy. It is, therefore, important to keep them under follow up in the form of endoscopic examination every three yearly and female patients are advised to attend cervical and breast screening programme.

\section{References}

1. Kopacova M, Tacheci I, Rejchrt S, Bures J. PeutzJeghers syndrome: diagnostic and therapeutic approach. World J Gastroenterol 2009; 15(43): 5397-5408.

2. Georgescue F, Stanescu L, Simionescu C, Georgescue I, Ionescu R, Florescu G, et al. PeutzJeghers syndrome: case report and literature review. Romanian J Morphol Embryol 2008, 49(2): 241-5.

3. Li Y, Zeng Q, Liao Z, Zhang G, Xiao R, Wen H. Peutz-Jeghers syndrome and family survey: a case report, Int J Clin Exp Pathol 2013; 6(5): 982-4.

4. Hafeez AA, Dahan A, Yasin MAK. Peutz-Jeghers Syndrome presented as intermittent gastric outlet obstruction and ileoileal intussusceptions. Sudan J Med Sci 2012; 7(2): 131-4.

5. Rodrigues GJ, et al. Intestinal intussusception and occlusion by small bowel polyps in the PeutzJeghers syndrome -management by combined intraoperative enteroscopy and resection through minimal enterostomy: case report. Rev Hosp Clin Fac Med S Paulo 2000; 55(6): 219-24.

6. Thakker HH, Joshi A, Deshpande A. PeutzJegher's syndrome presenting as jejunoileal intussusception in an adult male: a case report. Cases J 2009; 2: 8865.

7. Saxena PK, Arora RK, Singh HH. Peutz-Jeghers syndrome with unusual features (a case report). J Postgrad Med 1986; 32: 236.

8. Suzuki S, Hirasaki S, Ikeda F, Yumoto E, Yamane H, Matsubara M. Three cases of solitary PeutzJeghers-type hamartomatous polyp in the duodenum. World J Gastroenterol 2008; 14(6): 944-7.

9. Ananda D, Kannan N, Patil R, Sarath V, Gadagi JS. Diagnostic significance of Peutz-Jeghers syndrome: a case report with oral physician's perspective. Clin Cancer Investig J 2012; 1: 74-6.

10. Gazozai S, Sadiq S. Pautz-Jegher Syndrome: case report and review of literature. Biomedica 2005; 21.

11. Schreibman IR, Baker M, Amos C, McGarrity TJ. The hamartomatous polyposis syndromes: a clinical and molecular review. Am J Gastroenterol 2005; 100(2): 476-90. 